TTP18-023

\title{
Charm decays
}

\section{Stefan de Boer*}

Karlsruhe Institute of Technology

E-mail: stefan.boerakit.edu

The importance of charm decays in the context of flavor is motivated. A theory overview on recent developments in leptonic and semileptonic decays, hadronic two-body decays, and rare decays is presented. Standard Model (SM) predictions as well as tests of the SM are pointed out, e.g. how to discover direct $\mathrm{CP}$ violation in charm decays and how to search for physics beyond the SM.

Differences between rare charm and beauty decays are discussed.

The International Conference on B-Physics at Frontier Machines - BEAUTY2018

6-11 May, 2018

La Biodola, Elba Island, Italy

${ }^{*}$ Speaker. 


\section{Introduction}

Why talk about charm decays at the BEAUTY conference? We try to shed light on the link between beauty and charm in this proceedings. While $b$ decays are established to test the standard model (SM), charm decays are commonly considered to be blurred by non-calculable hadronic effects. These two members of the flavor family are driven by different dynamics, hence the way to search for physics beyond the SM (BSM) may point into different directions. In this sense, charm and beauty are complementary in BSM searches and both can learn from each other. One may compare the current situation of rare charm decays with that of the $b(\mathrm{ig})$ brother back twenty years, which resulted in the exciting times nowadays. Furthermore, charm is unique to test flavor in the up-type sector and allows for insights into QCD from a different perspective. Experimentally, the $b$ machines are also charm machines $[1,2,3,4]$. On the theoretical side one should be careful in adopting results from $b$ physics, e.g. the $1 / m_{c}$-counting is questionable and the short-long distance behavior is challenging. Additionally, the available phase space in charm decays is smaller, hence, e.g., decays into $\tau$ leptons are suppressed or even forbidden.

We present a theory overview on charm decays for a selection of recent developments in this field. Section 2 is an overview of current results of leptonic and semileptonic decays and determinations of the corresponding SM parameters. In section 3 we present the latest global fit to branching ratio data of hadronic two-body decays and outline how to test the SM with the potential to discover direct $\mathrm{CP}$ violation in charm decays. Rare decays, reviewed in section 4 , allow to search for BSM physics. Section 5 closes with the summary.

\section{Leptonic and semileptonic decays}

Leptonic and semileptonic $D \rightarrow l v$ and $D \rightarrow P l v$ decays, where $P$ denotes a pseudoscalar meson allow to determine several SM parameters. The decay into a $l=\tau$ lepton is kinematically suppressed/forbidden. The corresponding decay amplitudes read

$$
\begin{aligned}
\mathscr{A}(D \rightarrow l v) & \propto V_{c q}^{*}\left\langle 0\left|\bar{q} \gamma_{\mu} \gamma_{5} c\right| D\left(p^{\prime}\right)\right\rangle=V_{c q}^{*}\left[i p_{\mu}^{\prime} f_{D}\right], \\
\mathscr{A}(D \rightarrow P l v) & \propto V_{c q}^{*}\left\langle P(p)\left|q \gamma_{\mu} c\right| D\left(p^{\prime}\right)\right\rangle \\
& =V_{c q}^{*}\left[f_{+}\left(q^{2}\right)\left(\left(p^{\prime}+p\right)_{\mu}-\frac{m_{D}^{2}-m_{P}^{2}}{q^{2}} q_{\mu}\right)+f_{0}\left(q^{2}\right) \frac{m_{D}^{2}-m_{P}^{2}}{q^{2}} q_{\mu}\right],
\end{aligned}
$$

where $q^{2}=\left(p^{\prime}-p\right)^{2}$, the CKM factors $V_{c q}$, the decay constant $f_{D}$ and the form factors $f_{+, 0}\left(q^{2}\right)$. The amplitude (2.2) implies a lepton-mass-suppression of $f_{0}$ in charm decays, hence $f_{0}$ is presently barely accessible in experiments. The CKM factors are given as $V_{c d}=\lambda+\mathscr{O}\left(\lambda^{4}\right)$ and $V_{c s}=1-$ $\frac{\lambda^{2}}{2}+\mathscr{O}\left(\lambda^{4}\right)$ with $\lambda \sim 0.225$ in the Wolfenstein parametrization. Singly-Cabibbo suppressed $|\Delta c|=$ $|\Delta u|=1$ decays, that will be covered in the next sections, involve the products $V_{u d} V_{c d}^{*} \simeq-V_{u s} V_{c s}^{*}$. This approximate equality which reflects the GIM mechanism allows only small complex phases $\sim V_{u b} V_{c b}^{*}$.

As is evident from (2.1) and (2.2), experiments extract products of CKM factors with decay constants and form factors from leptonic and semileptonic decays, respectively. The individual quantities can then be obtained by either assuming CKM unitarity and the other CKM factors to be 


\begin{tabular}{ccc}
\hline & $f_{D}[\mathrm{MeV}]$ & $f_{D_{s}}[\mathrm{MeV}]$ \\
\hline HFLAV 2016 & $203.7(4.9)$ & $257.1(4.6)$ \\
FLAG 2016 & $212.15(1.45)$ & $248.83(1.27)$ \\
\hline
\end{tabular}

Table 1: Decay constants from experiments, assuming CKM unitarity, and $N_{f}=2+1+1$ LQCD calculations, summarized by HFLAV [5] and FLAG [6], respectively.

known or from a combination with, e.g., lattice QCD (LQCD) calculations. In practice, the CKM factors are most precisely known from fits to several observables employing CKM unitarity, hence we do not consider their extraction in the following.

The interplay of experiments and LQCD calculations in the determination of the decay constants is summarized in Table 1. The averaged results from experiments and LQCD calculations are compatible at $2 \sigma$ with competing but smaller uncertainties from the LQCD calculations. In addition, recent results from $N_{f}=2+1$ and $N_{f}=2+1+1$ calculations with individual uncertainties similar to the 2016 averaged ones are provided by RBC/UKQCD [7] and Fermilab Lattice/MILC [8], respectively. While lattice calculations need to include QED effects non-perturbatively in the future, also more precise experimental measurements are needed for further comparisons. Decay constants are also determined by QCD sum rule calculations, however, with larger uncertainties than the results collected in Table 1, e.g. $f_{D}=(208 \pm 10) \mathrm{MeV}$ and $f_{D_{s}}=(240 \pm 10) \mathrm{MeV}$ [9].

In Figure 1, we show the experimental average of the form factor $f_{+}$for $D \rightarrow(K, \pi)$. While ex-
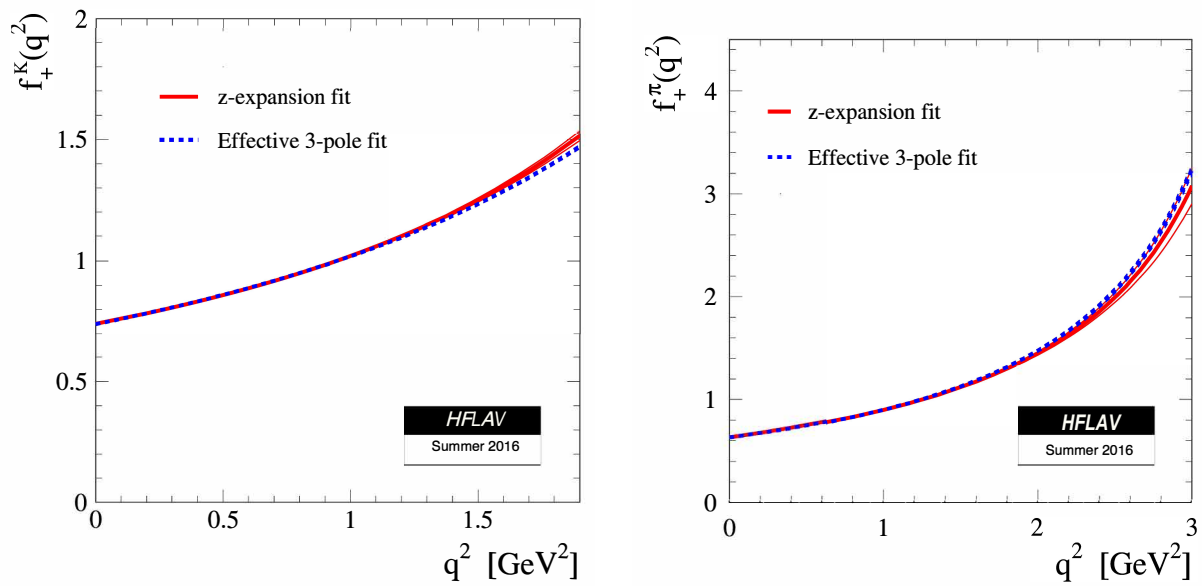

Figure 1: Form factor $f_{+}$for $D \rightarrow(K, \pi)$, averaged by HFLAV. Figure taken from [5].

periments precisely extract form factors at low $q^{2}$, form factors at high $q^{2}$ are accurately calculated on the lattice. In fact, the experimental results are in agreement with, e.g., the recent $N_{f}=2+1+1$ calculation provided by ETMC [10]. The interplay of experiments and LQCD calculations allows to determine form factors at the percent level precision and is also necessary in view of $f_{0}$ that is presently only accessible on the lattice.

Finally, we note that the form factors for $\Lambda_{c} \rightarrow N$, where $N$ is a nucleon, are available from a recent LQCD calculation [11]. 


\section{Hadronic two-body decays}

Hadronic two-body decays, $D \rightarrow P_{1} P_{2}$ with $P_{1,2} \in\{\pi, K\}$ have been studied extensively, see e.g. [12, 13, 14], including first attempts on the lattice [15]. Most of the branching ratios, their correlations and also CP-asymmetries are experimentally measured $[5,16]$. Theoretically, amplitudes for different decays can be related by the $S U(3)_{F}$-symmetry, allowing to test the SM. The amplitude for, e.g., a singly-Cabibbo suppressed decay is written as

$$
\mathscr{A}=\lambda_{s d} A_{s d}-\frac{\lambda_{b}}{2} A_{b}
$$

with $\lambda_{s d}=\frac{\lambda_{s}-\lambda_{d}}{2}$ and $\lambda_{q}=V_{c q}^{*} V_{u q}$. Recall that, while $\lambda_{b}$ can be neglected for branching ratios, it is the only source of CP violation in the SM. In the following, we summarize the global fit of branching ratios and the CP-asymmetry predictions given in the series of works $[17,18,19,20]$.

The framework consists of topological amplitudes with diagrammatic $S U(3)_{F}$-breaking and color-counting input. A global fit to branching ratio data reveals that the $S U(3)_{F}$-limit is excluded by more than $5 \sigma$, while $30 \% S U(3)_{F}$-breaking in the decay amplitudes is sufficient to describe the data [17]. Predictions on individual branching ratios are obtained as $\mathscr{B}\left(D_{s}^{+} \rightarrow K_{L} K^{+}\right)=$ $0.012_{-0.002}^{+0.006}$ at $3 \sigma \mathrm{CL}$ and $\mathscr{B}\left(D^{0} \rightarrow K_{L} \pi^{0}\right)<\mathscr{B}\left(D^{0} \rightarrow K_{S} \pi^{0}\right)$ with a significance of more than $4 \sigma[17]$.

For the direct $\mathrm{CP}$ violation, the corresponding asymmetry is written as

$$
a_{C P}^{\mathrm{dir}}=\operatorname{Im} \frac{\lambda_{b}}{\lambda_{s d}} \operatorname{Im} \frac{A_{b}}{A_{s d}} .
$$

Here, $\operatorname{Im} \frac{\lambda_{b}}{\lambda_{s d}} \simeq-6 \times 10^{-4}$ and $\left|A_{s d}\right|$ can be taken from the branching ratio fit. However, CPasymmetries require additional combinations of amplitudes that are not provided by a branching ratio fit. Nevertheless, these combinations can be eliminated by sum rules that correlate different $\mathrm{CP}$-asymmetries. To address charm CP violation, two strategies are given at hand: Firstly, the SM can be falsified with sum rules, or clean predictions. Secondly, direct CP violation can be discovered in charm, with large SM predictions being favored. An example for the first strategy is $A_{C P}\left(D^{+} \rightarrow \pi^{+} \pi^{0}\right) \simeq 0$ from isospin sum rules, e.g. [21], which is compatible with the recent Belle measurement $a_{C P}^{\mathrm{dir}}=+0.0231 \pm 0.0124 \pm 0.0023$ [22].

The discovery of direct CP violation is shown to be possible in $D^{0} \rightarrow K_{S} K_{S}$ decays with $\left|a_{C P}^{\mathrm{dir}}\right| \leq 1.1 . \%$ [19] that follows from sizable tree level exchange, and since $A_{s d}=0$ in the $S U(3)_{F^{-}}$ limit, while $A_{b} \neq 0$. Experimentally, $A_{C P}=-0.0002 \pm 0.0154$ is measured by Belle [23] with uncertainties being dominated by statistics as well as very recently LHCb measured $A_{C P}=0.042 \pm$ $0.034 \pm 0.010$ [24]. Direct CP violation can also be discovered in $D \rightarrow K_{S} K^{* 0}$ decays, $\left|a_{C P}^{\mathrm{dir}}\right| \leq 0.3 \%$ [20]. While the features of the $D^{0} \rightarrow K_{S} K_{S}$ decay also apply here, the decay $D \rightarrow K_{S} K^{* 0}$ is additionally experimentally favored, since charged tracks from prompt $K_{S} K^{* 0}$ decay can be identified, a Dalitz plot analysis may reveal regions with large strong phases to maximize the asymmetry, and no flavor tagging is required. No measurements for this asymmetry is presently available, though a first study is reported in [25]. 


\section{Rare decays}

Rare charm decays are very different from the analogous $b$ decays, nevertheless $|\Delta c|=|\Delta u|=1$ decays allow to test BSM physics in addition to and independent of their $b$ counterpart. We first review the anatomy of the SM, the background in BSM searches. The perturbative SM contribution is described by the Lagrangian $\mathscr{L}_{\text {eff }}^{\text {weak }} \sim \sum_{i} C_{i} P_{i}$ with Wilson coefficients $C_{i}$ and the corresponding operators, e.g.,

$$
\begin{aligned}
& P_{2(1)} \sim\left(\bar{u}_{L} \gamma_{\mu}\left(T^{a}\right) q_{L}\right)\left(\bar{q}_{L} \gamma^{\mu}\left(T^{a}\right) c_{L}\right), \\
& P_{7}^{(\prime)} \sim\left(\bar{u}_{L(R)} \sigma^{\mu v} c_{R(L)}\right) F_{\mu \nu}, \\
& P_{9(10)} \sim\left(\bar{u}_{L} \gamma_{\mu} c_{L}\right)\left(\bar{\ell} \gamma^{\mu}\left(\gamma_{5}\right) \ell\right),
\end{aligned}
$$

where the notation and the full set of operators can be found in, e.g., [26]. One characteristic of charm flavor-changing-neutral-current transitions is the two-step matching of the effective theory at the scales $m_{W}$ and $m_{b}$. Another one is related to the absence of heavy down-type quarks in loops, hence only two Wilson coefficients $\left(C_{1,2}\right)$ are nonzero at the scale $m_{W}$ and $C_{10} \simeq 0$ holds at the charm scale that is broken only by, e.g., electromagnetic effects. The effective Wilson coefficients, that include perturbative contributions which multiply the same matrix elements as the Wilson coefficients, are known at the same order as in $b$ physics $[27,28,29,30]$. The phenomenologically largest contributions to $C_{7,9}^{\text {eff }}$ result from two-loop QCD diagrams with insertions of $P_{1,2}$ [30], since the leading order contributions are generically suppressed by the GIM-mechanism due to closely degenerated light quarks in the loops. Note that the two-loop calculation [30] is valid for arbitrary momentum transfer and also for $b$ decays.

Switching from partons to hadrons, intermediate resonances evade the GIM-mechanism and dominate the branching ratios, though their contribution is uncertain, e.g. [31]. The different contributions to the branching ratios are summarized for $D^{+} \rightarrow \pi^{+} \mu^{+} \mu^{-}$in Figure 2. Charm decays

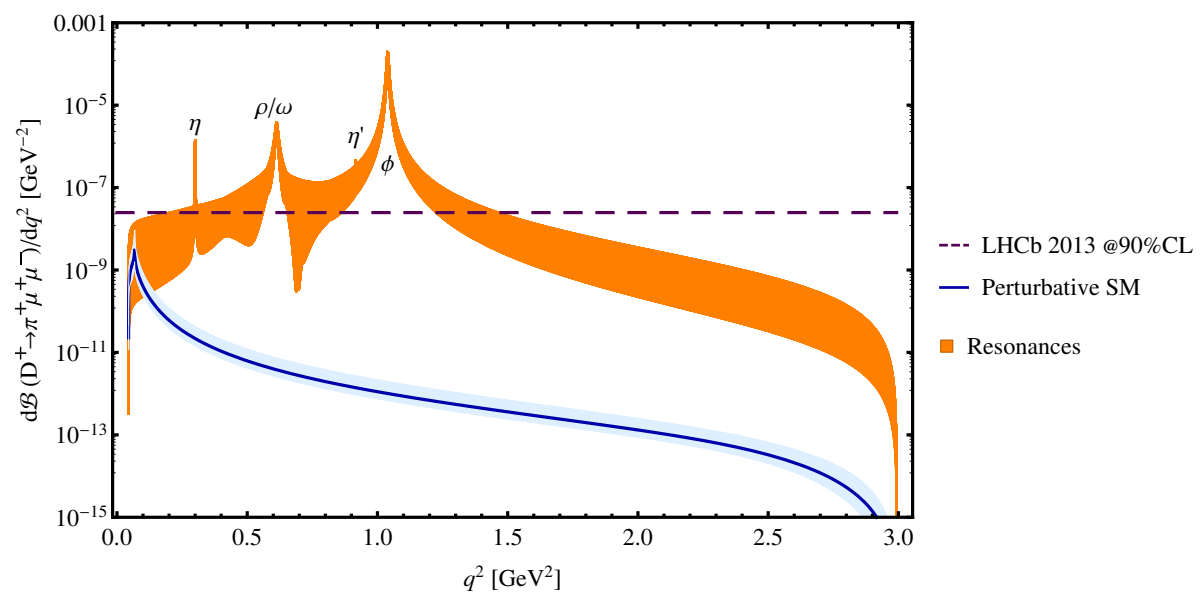

Figure 2: Differential branching ratio with respect to $q^{2}$ for $D^{+} \rightarrow \pi^{+} \mu^{+} \mu^{-}$. Figure is an update of [26].

may not look viable to search for BSM physics due to the large and uncertain SM background. On the other side, several unique SM features arise/persist, e.g., so-called "resonance-catalyzed" [32] 
observables as well as BSM sensitive observables due to the small SM weak phases and symmetries of QCD and QED. They allow to search for (heavy) BSM physics in different ways:

(a) Windows in branching ratios, e.g. the high $q^{2}$ region in Figure 2.

(b) Null test based on (approximate) SM symmetries.

(c) SM contribution extracted from SM-dominated modes as input for $S U(3)_{F}$-related, BSM sensitive modes.

Before illustrating these points, we emphasize to look into different decays and observables to probe the SM and sort BSM models. Figure 3 shows the sensitivity of the differential branching fraction for $D^{+} \rightarrow \pi^{+} \mu^{+} \mu^{-}$to different Wilson coefficients.

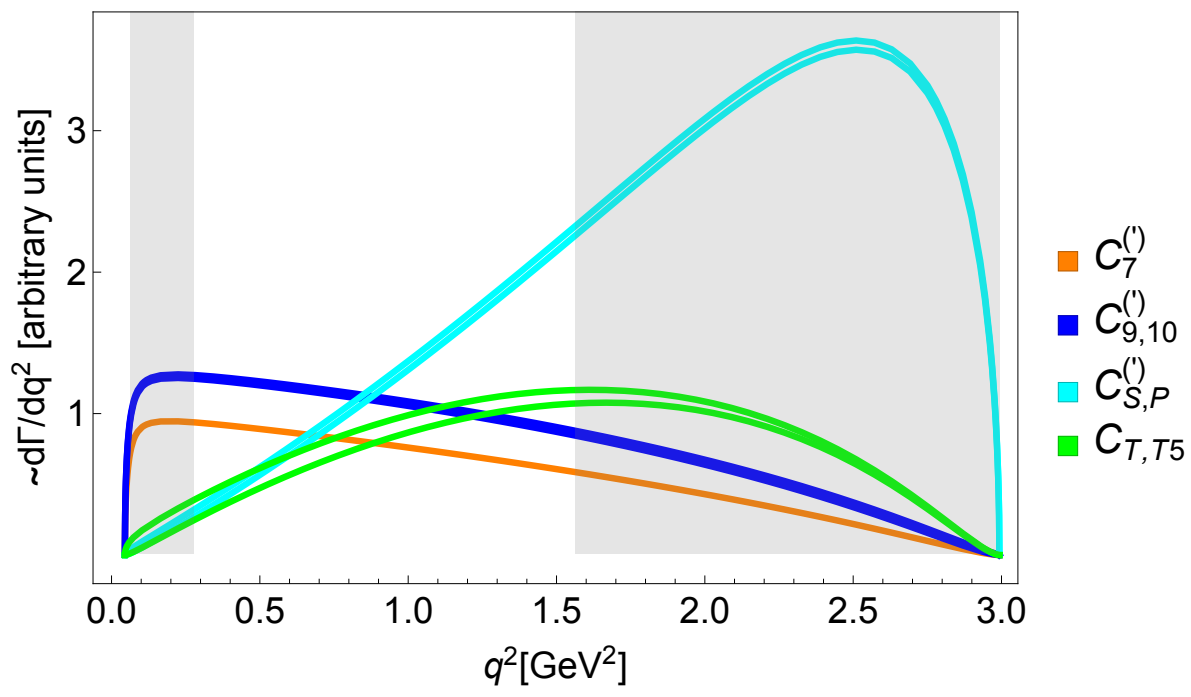

Figure 3: Comparison of short-distance spectrum sensitivities to different Wilson coefficient in $D^{+} \rightarrow$ $\pi^{+} \mu^{+} \mu^{-}$. Figure taken from [33].

Another example for (a) is the branching ratio of $D^{0} \rightarrow \mu^{+} \mu^{-}$. The present experimental upper limit $\mathscr{B}_{\text {exp }}<6.2 \times 10^{-9}$ by LHCb [34] sets the strongest constraints on the difference of (pseudo)scalar Wilson coefficients. While the SM branching ratio is commonly estimated orders of magnitude below $\mathscr{B}_{\exp }[35,36,37]$, a BSM-induced branching ratio close to the experimental limit is allowed within, e.g., two Higgs doublet and leptoquark models [26, 33, 35, 37, 38, 39, 40]. The decay into electrons is, on the other hand, helicity suppressed and diluted by misidentification from $\mathscr{O}\left(\alpha m_{D}^{2} / m_{e}^{2}\right)$ enhanced $D^{0} \rightarrow e^{+} e^{-} \gamma$ decays with soft photons [28]. The helicity suppression is lifted for $D^{0^{*}} \rightarrow e^{+} e^{-}$decays, however $D^{0^{*}}$ decays strongly/electromagnetically. Note that the decay $D^{0^{*}} \rightarrow \mu^{+} \mu^{-}$is also diluted by misidentification from $D^{0^{*}} \rightarrow \pi^{+} \pi^{-}$decays. As explored in [41], $e^{+} e^{-} \rightarrow D^{0^{*}}$ decays evade these complications. The SM branching ratio of $e^{+} e^{-} \rightarrow D^{0^{*}}$ is predicted to be $\mathscr{B}_{\mathrm{SM}} \sim 10^{-18}$, while in $Z^{\prime}$ models $\mathscr{B}_{Z^{\prime}}<2.5 \times 10^{-11}$ [41]. A measurement would probe (axial)vector Wilson coefficients, not accessible in $D^{0} \rightarrow l l$ decays.

Generically, for $c \rightarrow u \ell \ell^{(\prime)}$ induced decays, e.g. $D \rightarrow P \ell \ell$ and $D \rightarrow P P \ell \ell$, SM CP-asymmetries are small, $A_{C P}^{\mathrm{SM}} \sim \frac{\mathrm{Im} \lambda_{b}}{\lambda_{s}} \sim 10^{-3}$. Further SM null test are given in terms of angular observables, 


\begin{tabular}{ccc}
\hline model & $A_{C P}$ & $A_{F B}$ \\
\hline Leptoquark models & $\gtrsim A_{C P}^{\mathrm{SM}}$ & $\lesssim 8 \times 10^{-1}$ \\
Little Higgs model & $\lesssim \mathscr{O}\left(10^{-3}\right)$ & $\lesssim \mathscr{O}\left(5 \times 10^{-3}\right)$ \\
Minimal SUSY SM & $\lesssim \mathscr{O}\left(10^{-3}\right)$ & $\lesssim \mathscr{O}\left(10^{-1}\right)$ \\
Up vector-like quark singlet & - & $\lesssim 10^{-3}$ \\
Warped extra dimension & $\lesssim \mathscr{O}\left(10^{-2}\right)$ & $\lesssim \mathscr{O}\left(5 \times 10^{-2}\right)$ \\
Z' boson & - & $\sim 0$ \\
\hline SM & $<\mathscr{O}\left(10^{-3}\right)$ & $\sim 0$ \\
\hline
\end{tabular}

Table 2: CP asymmetry and forward-backward asymmetry within BSM models. Table is a compilation of the results given in $[26,33,35,39,40,48,49,50,51,52,53,54,55,56,57,58,59]$.

e.g. the dilepton forward-backward asymmetry, which involve $C_{10}$, (pseudo)scalar and tensor Wilson coefficients and are suppressed in the SM. Additionally, the SM can be testes with decays into different leptons. Lepton-universality, probed by the ratio of muons/electrons, holds up to percentage corrections in the SM [33]. Experimentally, decays into electrons and muons are measured in different experiments, e.g. [42, 43] employed different cuts for $D \rightarrow P P l l$ decays. Lepton-flavorviolating decays, also from quarkonia, are absent in the SM [26, 35, 44, 45], hence are further null tests of the SM. Decays into neutrinos which vanish in the SM and also probe dark matter $[26,35,39,46]$ are bounded by Belle [47]. Predictions for the CP-asymmetry and the forwardbackward asymmetry within BSM models are compiled in Table 2, ranging from "SM-like" to "within reach of the next measurement".

The following example of a "resonance-catalyzed" [32] CP-asymmetry comprises several of the just discussed qualities. Consider a scalar leptoquark (with quantum numbers $(3,3,-1 / 3)$ ), supplement a flavor pattern (inspired by $b$ decays [60]) and respect constraints from Kaon decays (due to $S U(2)_{L}$ couplings). This recipe results in Figure 4, illustrating that the CP-asymmetry around resonances probes the Wilson coefficient $\operatorname{Im} C_{9}^{\mathrm{BSM}}$, independent of strong phases.

Finally, we discuss radiative $c \rightarrow u \gamma$ induced decays. Again, branching ratios are dominated by uncertain long-distance effects $[28,61,62,63,64,65,66,67,68,69]$. On the other hand, the decay $B_{c} \rightarrow B_{u}^{*} \gamma$ and the ratio $\Gamma\left(D^{0} \rightarrow \rho^{0} \gamma\right) / \Gamma\left(D^{0} \rightarrow \omega \gamma\right)$ are sensitive to BSM physics [70, 71]. The $\mathrm{SM}$ prediction for $\mathrm{CP}$ asymmetries is, again, $A_{C P}^{\mathrm{SM}}<\mathscr{O}\left(10^{-3}\right)$, while in BSM models $A_{C P}^{\mathrm{BSM}} \lesssim 10 \%$ $[67,72,73]$. Here, the first experimental measurement of $A_{C P}^{\exp }\left(D^{0} \rightarrow \rho^{0} \gamma\right)=0.056 \pm 0.152$, where the statistical uncertainty dominates, is obtained by Belle [74].

A feature of radiative decays is an observable photon polarization, the ratio of right/left-handed currents $\left(C_{7}^{\prime} / C_{7}\right)$. The photon polarization can be probed in the following ways.

- Time-dependent analysis: Relate the SM-dominated decay $\bar{D}^{0} \rightarrow \bar{K}^{*}{ }^{0} \gamma$ to the decays $\bar{D}^{0} \rightarrow$ $\left(\rho^{0} / \omega, \phi\right) \gamma$ using data and $S U(3)_{F}$-symmetry and extract the BSM contribution $[73,75]$.

- The up-down asymmetry of the decay $D \rightarrow \bar{K}_{1}(\rightarrow \bar{K} \pi \pi) \gamma$ is independent of strong phases between $C_{7}$ and $C_{7}^{\prime}$ and heavier resonances are phase space suppressed, but $D$-tagging is required [75]. 


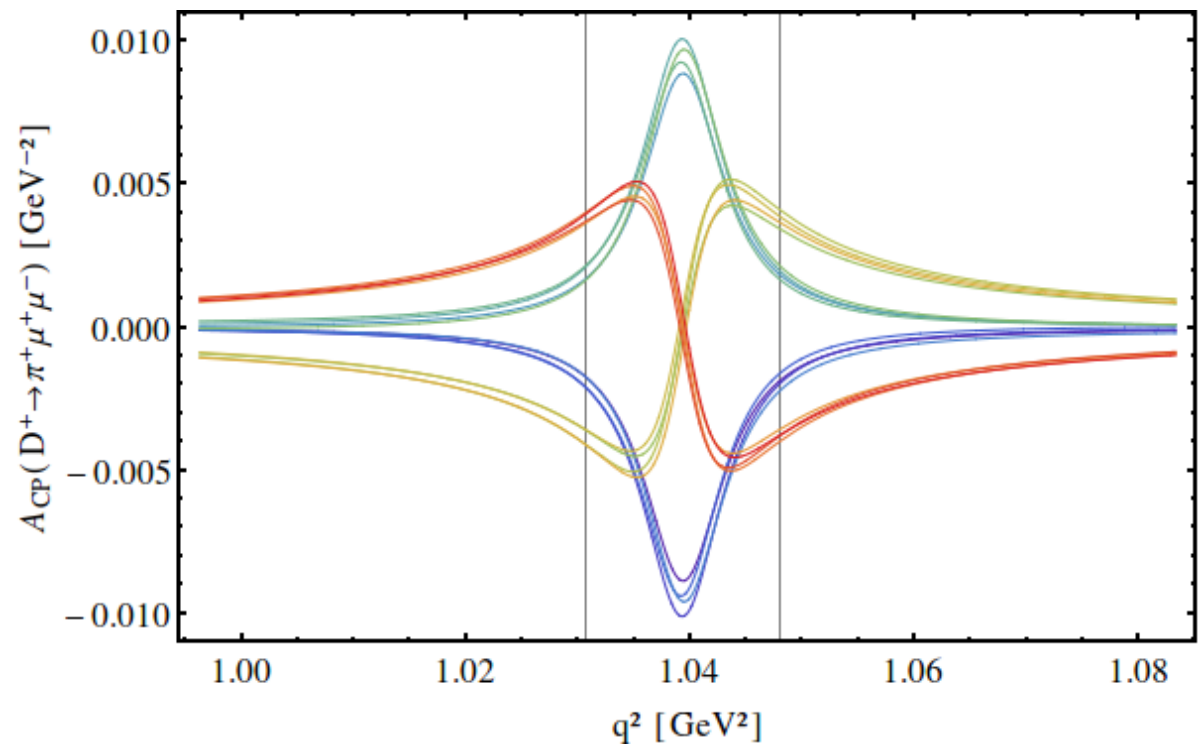

Figure 4: Resonance-catalyzed CP-asymmetry for a scalar leptoquark model, around the $\phi$ resonance, normalized to the shown bin and for the strong phases $(\pi / 2, \pi, 0,3 / 2 \pi)$. Figure taken from [26].

- The photon forward-backward asymmetry in the decay $\Lambda_{c} \rightarrow p \gamma$ can be measured at future colliders [67].

\section{Summary}

Charm decays allow for a wide analysis of the SM and beyond. On the one hand, SM parameters, namely decay constants and form factors, are precisely known from leptonic and semileptonic decays. Recent experiments and LQCD computations challenge each other with competing uncertainties. On the other hand, hadronic two-body decays allow for global fits to branching ratio data and to test the $S U(3)_{F}$-symmetry. Direct CP violation of charm decays in the SM can be discover in $D^{0} \rightarrow K_{S} K_{S}$ and $D \rightarrow K_{S} K^{* 0}$ decays with $\mathrm{SM} \mathrm{CP}$ asymmetries $\lesssim 1 \%$.

Rare charm decays allow to uniquely probe the SM and BSM physics with different decays and observables - despite branching ratios being dominated by long-distance effects. Examples are CP-asymmetries and angular observables in semileptonic decays, decays into different leptons as SM null test and connections between different radiative decays. While BSM physics may link flavor sectors, one should not forget that charm decays may also improve our understanding of QCD and check theoretical frameworks. Several experiments and theoretical works are ongoing, e.g. on $D \rightarrow P P l l$ decays $[76,77]$.

\section{Acknowledgements}

I thank the organizers for the invitation and the wonderful conference. I am grateful to Ulrich Nierste, Stefan Schacht and Michele Della Morte for valuable discussion. This work has been supported by the BMBF under contract no. 05H15VKKB1. I acknowledge the DAAD for financial support to attend the conference. 


\section{References}

[1] L. Li (On behalf of the Belle Collaboration), "Charm physics at B-factories," these proceedings

[2] M. Williams, On behalf of the LHCb Collaboration, "Charm mixing and CP violation at LHCb," these proceedings

[3] G. Mezzadri On behalf of the BESIII Collaboration, "Semi-leptonic and leptonic $D^{0(+)}$ and $D_{s}$ decays at BESIII," these proceedings

[4] A. Di Canto on behalf of the LHCb Collaboration, "Rare charm and strange decays," these proceedings

[5] Y. Amhis et al. [HFLAV Collaboration], Eur. Phys. J. C 77, no. 12, 895 (2017) doi:10.1140/epjc/s10052-017-5058-4 [arXiv:1612.07233 [hep-ex]].

[6] S. Aoki et al., Eur. Phys. J. C 77, no. 2, 112 (2017) doi:10.1140/epjc/s10052-016-4509-7 [arXiv:1607.00299 [hep-lat]].

[7] P. A. Boyle, L. Del Debbio, A. Jüttner, A. Khamseh, F. Sanfilippo and J. T. Tsang, JHEP 1712, 008 (2017) doi:10.1007/JHEP12(2017)008 [arXiv:1701.02644 [hep-lat]].

[8] A. Bazavov et al., arXiv:1712.09262 [hep-lat].

[9] Z. G. Wang, Eur. Phys. J. C 75, 427 (2015) doi:10.1140/epjc/s10052-015-3653-9 [arXiv:1506.01993 [hep-ph]].

[10] V. Lubicz et al. [ETM Collaboration], Phys. Rev. D 96, no. 5, 054514 (2017) doi:10.1103/PhysRevD.96.054514 [arXiv:1706.03017 [hep-lat]].

[11] S. Meinel, Phys. Rev. D 97, no. 3, 034511 (2018) doi:10.1103/PhysRevD.97.034511 [arXiv:1712.05783 [hep-lat]].

[12] J. Brod, Y. Grossman, A. L. Kagan and J. Zupan, JHEP 1210, 161 (2012) doi:10.1007/JHEP10(2012)161 [arXiv:1203.6659 [hep-ph]].

[13] G. Hiller, M. Jung and S. Schacht, Phys. Rev. D 87, no. 1, 014024 (2013) doi:10.1103/PhysRevD.87.014024 [arXiv:1211.3734 [hep-ph]].

[14] A. Khodjamirian and A. A. Petrov, Phys. Lett. B 774, 235 (2017) doi:10.1016/j.physletb.2017.09.070 [arXiv:1706.07780 [hep-ph]].

[15] M. T. Hansen and S. R. Sharpe, Phys. Rev. D 86, 016007 (2012) doi:10.1103/PhysRevD.86.016007 [arXiv:1204.0826 [hep-lat]].

[16] C. Patrignani et al. [Particle Data Group], Chin. Phys. C 40, no. 10, 100001 (2016). doi:10.1088/1674-1137/40/10/100001

[17] S. Müller, U. Nierste and S. Schacht, Phys. Rev. D 92, no. 1, 014004 (2015) doi:10.1103/PhysRevD.92.014004 [arXiv:1503.06759 [hep-ph]].

[18] S. Müller, U. Nierste and S. Schacht, Phys. Rev. Lett. 115, no. 25, 251802 (2015) doi:10.1103/PhysRevLett.115.251802 [arXiv:1506.04121 [hep-ph]].

[19] U. Nierste and S. Schacht, Phys. Rev. D 92, no. 5, 054036 (2015) doi:10.1103/PhysRevD.92.054036 [arXiv:1508.00074 [hep-ph]].

[20] U. Nierste and S. Schacht, Phys. Rev. Lett. 119, no. 25, 251801 (2017) doi:10.1103/PhysRevLett.119.251801 [arXiv:1708.03572 [hep-ph]]. 
[21] Y. Grossman, A. L. Kagan and J. Zupan, Phys. Rev. D 85, 114036 (2012) doi:10.1103/PhysRevD.85.114036 [arXiv:1204.3557 [hep-ph]].

[22] V. Babu et al. [Belle Collaboration], Phys. Rev. D 97, no. 1, 011101 (2018) doi:10.1103/PhysRevD.97.011101 [arXiv:1712.00619 [hep-ex]].

[23] N. Dash et al., Phys. Rev. Lett. 119, no. 17, 171801 (2017) doi:10.1103/PhysRevLett.119.171801 [arXiv:1705.05966 [hep-ex]].

[24] R. Aaij et al. [LHCb Collaboration], arXiv:1806.01642 [hep-ex].

[25] R. Aaij et al. [LHCb Collaboration], Phys. Rev. D 93, no. 5, 052018 (2016) doi:10.1103/PhysRevD.93.052018 [arXiv:1509.06628 [hep-ex]].

[26] S. de Boer and G. Hiller, Phys. Rev. D 93, no. 7, 074001 (2016) doi:10.1103/PhysRevD.93.074001 [arXiv:1510.00311 [hep-ph]].

[27] C. Greub, T. Hurth, M. Misiak and D. Wyler, Phys. Lett. B 382, 415 (1996) doi:10.1016/0370-2693(96)00694-6 [hep-ph/9603417].

[28] S. Fajfer, P. Singer and J. Zupan, Eur. Phys. J. C 27, 201 (2003) doi:10.1140/epjc/s2002-01090-5 [hep-ph/0209250].

[29] S. de Boer, B. Müller and D. Seidel, JHEP 1608, 091 (2016) doi:10.1007/JHEP08(2016)091 [arXiv:1606.05521 [hep-ph]].

[30] S. de Boer, Eur. Phys. J. C 77, no. 11, 801 (2017) doi:10.1140/epjc/s10052-017-5364-x [arXiv:1707.00988 [hep-ph]].

[31] T. Feldmann, B. Müller and D. Seidel, JHEP 1708, 105 (2017) doi:10.1007/JHEP08(2017)105 [arXiv:1705.05891 [hep-ph]].

[32] S. Fajfer and N. Košnik, Phys. Rev. D 87, no. 5, 054026 (2013) doi:10.1103/PhysRevD.87.054026 [arXiv:1208.0759 [hep-ph]].

[33] S. Fajfer and N. Košnik, Eur. Phys. J. C 75, no. 12, 567 (2015) doi:10.1140/epjc/s10052-015-3801-2 [arXiv:1510.00965 [hep-ph]].

[34] R. Aaij et al. [LHCb Collaboration], Phys. Lett. B 725, 15 (2013) doi:10.1016/j.physletb.2013.06.037 [arXiv:1305.5059 [hep-ex]].

[35] G. Burdman, E. Golowich, J. L. Hewett and S. Pakvasa, Phys. Rev. D 66, 014009 (2002) doi:10.1103/PhysRevD.66.014009 [hep-ph/0112235].

[36] S. Fajfer, P. Singer and J. Zupan, Phys. Rev. D 64, 074008 (2001) doi:10.1103/PhysRevD.64.074008 [hep-ph/0104236].

[37] A. Paul, I. I. Bigi and S. Recksiegel, Phys. Rev. D 82, 094006 (2010) Erratum: [Phys. Rev. D 83, 019901 (2011)] doi:10.1103/PhysRevD.83.019901, 10.1103/PhysRevD.82.094006 [arXiv:1008.3141 [hep-ph]].

[38] E. Golowich, J. Hewett, S. Pakvasa and A. A. Petrov, Phys. Rev. D 79, 114030 (2009) doi:10.1103/PhysRevD.79.114030 [arXiv:0903.2830 [hep-ph]].

[39] A. Paul, A. De La Puente and I. I. Bigi, Phys. Rev. D 90, no. 1, 014035 (2014) doi:10.1103/PhysRevD.90.014035 [arXiv:1212.4849 [hep-ph]].

[40] R. M. Wang, J. H. Sheng, J. Zhu, Y. Y. Fan and Y. G. Xu, Int. J. Mod. Phys. A 30, no. 12, 1550063 (2015) doi:10.1142/S0217751X15500633 [arXiv:1409.0181 [hep-ph]]. 
[41] A. Khodjamirian, T. Mannel and A. A. Petrov, JHEP 1511, 142 (2015) doi:10.1007/JHEP11(2015)142 [arXiv:1509.07123 [hep-ph]].

[42] R. Aaij et al. [LHCb Collaboration], Phys. Rev. Lett. 119, no. 18, 181805 (2017) doi:10.1103/PhysRevLett.119.181805 [arXiv:1707.08377 [hep-ex]].

[43] M. Ablikim et al. [BESIII Collaboration], Phys. Rev. D 97, no. 7, 072015 (2018) doi:10.1103/PhysRevD.97.072015 [arXiv:1802.09752 [hep-ex]].

[44] D. E. Hazard and A. A. Petrov, Phys. Rev. D 94, no. 7, 074023 (2016) doi:10.1103/PhysRevD.94.074023 [arXiv:1607.00815 [hep-ph]].

[45] D. E. Hazard and A. A. Petrov, arXiv:1711.05314 [hep-ph].

[46] A. Badin and A. A. Petrov, Phys. Rev. D 82, 034005 (2010) doi:10.1103/PhysRevD.82.034005 [arXiv:1005.1277 [hep-ph]].

[47] Y.-T. Lai et al. [Belle Collaboration], Phys. Rev. D 95, no. 1, 011102 (2017) doi:10.1103/PhysRevD.95.011102 [arXiv:1611.09455 [hep-ex]].

[48] S. Fajfer, S. Prelovsek and P. Singer, Phys. Rev. D 58, 094038 (1998) doi:10.1103/PhysRevD.58.094038 [hep-ph/9805461].

[49] S. Fajfer, S. Prelovsek and P. Singer, Phys. Rev. D 64, 114009 (2001) doi:10.1103/PhysRevD.64.114009 [hep-ph/0106333].

[50] S. Fajfer and S. Prelovsek, Phys. Rev. D 73, 054026 (2006) doi:10.1103/PhysRevD.73.054026 [hep-ph/0511048].

[51] S. Fajfer and S. Prelovsek, Conf. Proc. C 060726, 811 (2006) [hep-ph/0610032].

[52] S. Fajfer, N. Kosnik and S. Prelovsek, Phys. Rev. D 76, 074010 (2007) doi:10.1103/PhysRevD.76.074010 [arXiv:0706.1133 [hep-ph]].

[53] S. Fajfer and N. Kosnik, Phys. Rev. D 79, 017502 (2009) doi:10.1103/PhysRevD.79.017502 [arXiv:0810.4858 [hep-ph]].

[54] A. Paul, I. I. Bigi and S. Recksiegel, Phys. Rev. D 83, 114006 (2011) doi:10.1103/PhysRevD.83.114006 [arXiv:1101.6053 [hep-ph]].

[55] I. I. Bigi and A. Paul, JHEP 1203, 021 (2012) doi:10.1007/JHEP03(2012)021 [arXiv:1110.2862 [hep-ph]].

[56] C. Delaunay, J. F. Kamenik, G. Perez and L. Randall, JHEP 1301, 027 (2013) doi:10.1007/JHEP01(2013)027 [arXiv:1207.0474 [hep-ph]].

[57] L. Cappiello, O. Cata and G. D'Ambrosio, JHEP 1304, 135 (2013) doi:10.1007/JHEP04(2013)135 [arXiv:1209.4235 [hep-ph]].

[58] X. D. Guo, X. Q. Hao, H. W. Ke, M. G. Zhao and X. Q. Li, Chin. Phys. C 41, no. 9, 093107 (2017) doi:10.1088/1674-1137/41/9/093107 [arXiv:1703.08799 [hep-ph]].

[59] S. Sahoo and R. Mohanta, Eur. Phys. J. C 77, no. 5, 344 (2017) doi:10.1140/epjc/s10052-017-4888-4 [arXiv:1705.02251 [hep-ph]].

[60] I. de Medeiros Varzielas and G. Hiller, JHEP 1506, 072 (2015) doi:10.1007/JHEP06(2015)072 [arXiv:1503.01084 [hep-ph]].

[61] G. Burdman, E. Golowich, J. L. Hewett and S. Pakvasa, Phys. Rev. D 52, 6383 (1995) doi:10.1103/PhysRevD.52.6383 [hep-ph/9502329]. 
[62] A. Khodjamirian, G. Stoll and D. Wyler, Phys. Lett. B 358, 129 (1995) doi:10.1016/0370-2693(95)00972-N [hep-ph/9506242].

[63] S. Fajfer and P. Singer, Phys. Rev. D 56, 4302 (1997) doi:10.1103/PhysRevD.56.4302 [hep-ph/9705327].

[64] S. Fajfer, S. Prelovsek and P. Singer, Eur. Phys. J. C 6, 471 (1999) doi:10.1007/s100520050356, 10.1007/s100529800914 [hep-ph/9801279].

[65] S. Prelovsek and D. Wyler, Phys. Lett. B 500, 304 (2001) doi:10.1016/S0370-2693(01)00077-6 [hep-ph/0012116].

[66] M. Dimou, J. Lyon and R. Zwicky, Phys. Rev. D 87, no. 7, 074008 (2013) doi:10.1103/PhysRevD.87.074008 [arXiv:1212.2242 [hep-ph]].

[67] S. de Boer and G. Hiller, JHEP 1708 (2017) 091 doi:10.1007/JHEP08(2017)091 [arXiv:1701.06392 [hep-ph]].

[68] A. Biswas, S. Mandal and N. Sinha, arXiv:1702.05059 [hep-ph].

[69] J. M. Dias, V. R. Debastiani, J. J. Xie and E. Oset, Chin. Phys. C 42, no. 4, 043106 (2018) doi:10.1088/1674-1137/42/4/043106 [arXiv:1711.09924 [hep-ph]].

[70] S. Fajfer, S. Prelovsek and P. Singer, Phys. Rev. D 59, 114003 (1999) Erratum: [Phys. Rev. D 64, 099903 (2001)] doi:10.1103/PhysRevD.59.114003, 10.1103/PhysRevD.64.099903 [hep-ph/9901252].

[71] S. Fajfer, S. Prelovsek, P. Singer and D. Wyler, Phys. Lett. B 487, 81 (2000) doi:10.1016/S0370-2693(00)00731-0 [hep-ph/0006054].

[72] G. Isidori and J. F. Kamenik, Phys. Rev. Lett. 109, 171801 (2012) doi:10.1103/PhysRevLett.109.171801 [arXiv:1205.3164 [hep-ph]].

[73] J. Lyon and R. Zwicky, arXiv:1210.6546 [hep-ph].

[74] A. Abdesselam et al. [Belle Collaboration], Phys. Rev. Lett. 118, no. 5, 051801 (2017) doi:10.1103/PhysRevLett.118.051801 [arXiv:1603.03257 [hep-ex]].

[75] S. de Boer and G. Hiller, Eur. Phys. J. C 78, no. 3, 188 (2018) doi:10.1140/epjc/s10052-018-5682-7 [arXiv:1802.02769 [hep-ph]].

[76] S. de Boer and G. Hiller, arXiv:1805.08516 [hep-ph].

[77] R. Aaij et al. [LHCb Collaboration], arXiv:1806.10793 [hep-ex]. 